\title{
Resolution scaling in noncontact scanning impedance imaging
}

Benjamin C. Green

benjamin.green@asu.edu

Tao Shang

Jacey C. Morine

Hongze Liu

Stephen M. Schultz

See next page for additional authors

Follow this and additional works at: https://scholarsarchive.byu.edu/facpub

Part of the Electrical and Computer Engineering Commons

\section{Original Publication Citation}

Green, Benjamin C., Tao Shang, Jacey C. Morine, Hongze Liu, Stephen M. Schultz, Travis E. Oliphant, and Aaron R. Hawkins. "Resolution scaling in noncontact scanning impedance imaging." Review of Scientific Instruments 75 (24): 461-4614

\section{BYU ScholarsArchive Citation}

Green, Benjamin C.; Shang, Tao; Morine, Jacey C.; Liu, Hongze; Schultz, Stephen M.; Oliphant, Travis E.; and Hawkins, Aaron R., "Resolution scaling in noncontact scanning impedance imaging" (2004). Faculty Publications. 410.

https://scholarsarchive.byu.edu/facpub/410

This Peer-Reviewed Article is brought to you for free and open access by BYU ScholarsArchive. It has been accepted for inclusion in Faculty Publications by an authorized administrator of BYU ScholarsArchive. For more information, please contact ellen_amatangelo@byu.edu. 


\section{Authors}

Benjamin C. Green, Tao Shang, Jacey C. Morine, Hongze Liu, Stephen M. Schultz, Travis E. Oliphant, and Aaron R. Hawkins 


\title{
Resolution scaling in noncontact scanning impedance imaging
}

\author{
Benjamin C. Green, Tao Shang, Jacey C. Morine, Hongze Liu, Stephen M. Schultz, \\ Travis E. Oliphant, and Aaron R. Hawkins ${ }^{\text {a) }}$ \\ Electrical and Computer Engineering Department, Brigham Young University, 459 Clyde Building, \\ Provo, Utah 84602
}

(Received 15 July 2004; accepted 23 August 2004; published 29 October 2004)

\begin{abstract}
Noncontact scanning impedance imaging has been presented as a method to provide high resolution, high contrast images for a variety of material systems. This technique combines electrical impedance measurements with very high resolution scanning. This article reports on efforts to scale this technique down to the very important single micron range and reveals measurements for both thick and thin samples with a measured minimum resolution below $30 \mu \mathrm{m}$. A design for a shielded impedance probe applicable to this process is outlined and probes of several different sizes were made and tested. Fabrication of these impedance probes is explained and a testing methodology to characterize the probes' imaging capability is outlined. Measured results are reported and compared to a predictive model based on image blurring. Two-dimensional impedance images of objects have also been made indicating good image contrast and high resolution. Based on measured data and the model, scaling down to submicron resolution dimensions should be possible given small diameter probes and thin test samples. (C) 2004 American Institute of Physics. [DOI: 10.1063/1.1808127]
\end{abstract}

\section{INTRODUCTION}

There are a variety of current methods that go beyond standard optical microscopy to achieve micron-scale imaging: atomic force microscopy (AFM), scanning electron microscopy (SEM), near fields scanning optical microscopy (NSOM), etc. These methods use very fine scanning to produce very high-resolution images but are primarily used for profiling surfaces. Even though such profiles provide useful structural information, often they cannot distinguish between different materials and are of limited use for measuring composition through a given material thickness. In an attempt to provide more composition information, some of these scanning imaging methods have been altered to accentuate image contrast, for example, SEM imaging incorporating electron backscatter $^{1}$ and NSOM using fluorescent imaging. ${ }^{2}$ In addition, AFM has been used to measure electrical properties for a material in direct contact with a sharp probe tip. ${ }^{3}$

None of the above modification, however, fully addresses the need for a noncontact measurement technique that could provide high-contrast, high-resolution images taken through a sample where noncontact refers to no direct physical contact between a probe and the sample. All of these requirements are fulfilled using a technique called noncontact scanning impedance imaging (SII). ${ }^{4}$ Rather than attempting to incorporate higher material contrast into existing microscale imaging systems, SII uses the contrast that is inherent in electrical impedance combined with the highresolution and imaging capabilities possible with scanning. Measurements are done with the sample immersed in a conducting solution eliminating the need to account for the complexity of contact resistance inherent in surface contact techniques. This is especially important for soft tissues or

${ }^{\text {a)} E l e c t r o n i c ~ m a i l: ~ h a w k i n s @ e e . b y u . e d u ~}$ materials with large impedances where contact resistance can vary significantly with surface conditions of both the probe and the material.

The scanning impedance imaging technique is best applied to uniformly thick samples that exhibit a change in impedance across a given area. Achievable resolution will be very dependent on sample thickness. The same techniques used to prepare thin samples for electron microscopy could of course be used to prepare samples for SII. There are a large variety of material systems that could potentially be explored. Two of the most obvious are very large scale integrated semiconductor circuitry and biological cells. Semiconductor circuits have very high impedance contrasts over their surface area and submicron scale SII would allow for them to be imaged through different layers of semiconductor, insulator, or metal. SII is attractive for biological tissues because it can be done in a conducting aqueous solution, and tissues can then be imaged in vitro. A high-resolution electrical impedance image of biological tissues could reveal a great deal of information due to the biologically significant behavior of electrically active ions (e.g., sodium, potassium, chlorine, and calcium), polar molecules (e.g., water), and inhomogeneous charge distributions (e.g., most proteins).

In fact, electrical impedance has been studied as a source of biomedically relevant contrast for literally decades. As early as 1923, Grant found that cerebral gliomas showed roughly half the resistance (real part of impedance) of normal tissue. ${ }^{5}$ Similar observations applied to neoplastic growth in the breast have led to several related methods aimed to assist in the detection and diagnosis of cancer. ${ }^{6}$ Biological tissues contain a variety of ions, polar molecules, and inhomogeneous charge distributions that can affect the way electromagnetic fields interact with the material even on a very small scale. This physical fact provides for the possi- 
bility of obtaining a wealth of information about both normal and pathological tissue using some form of electromagnetic imaging outside of the usual optical imaging frequencies. For example, aside from the experiments showing higher conductivity in tumors, Geddes and Baker have found resistivity contrast of human tissues to be about 200:1 in human tissues at low frequencies. ${ }^{7}$

These biomedical results, however, were obtained using the technique of electrical impedance tomography which has very low resolution, limiting its application. Truly significant results would result if impedance imaging at multiple frequencies could be done on a micron and submicron scale. For biological research, this would allow inter- and intracellular measurements of both the conductivity (how easily charges move) and the dielectric permeability (how easily polar molecules reorient) of cellular tissue, yielding greater insight into cellular function and makeup. For material science research, this resolution would allow for imaging on the order of important grain boundary sizes. And for electronics research, imaging modern small-scale semiconductor devices would be possible.

A report of noncontact scanning impedance imaging ${ }^{4}$ cited a measured minimum resolution of $150 \mu \mathrm{m}$ for relatively thick samples. This article reports on efforts to scale this technique down to the very important single micron range and reveals measurements for both thick and thin samples with a measured minimum resolution below $30 \mu \mathrm{m}$. Fabrication of small impedance probes and testing methodology is explained in Sec. II. Measured results are reported and compared to a predictive model based on image blurring in Sec. III. Prospects and predictions for further resolution scaling are also discussed in this section.

\section{IMAGING SYSTEM}

Electrical impedance (or admittance) refers in general to the relationship between both conduction current and displacement current from an imposed electric field. In linear, isotropic materials, the central equation of electrical impedance imaging at any frequency $\omega$ is

$$
\nabla \cdot(\sigma \mathbf{E})=0,
$$

where $\sigma=\sigma^{\prime}+j \omega \varepsilon$ is the admittance, $\sigma^{\prime}$ is the conductivity, $\varepsilon$ is the dielectric constant of the material, and $\mathbf{E}$ is the electric field in the material. The general idea of electrical impedance imaging is to recover the distribution of $\sigma$ in the region of interest using electrical sources at the boundary. The preliminary work reported in Sec. III reports on only recovering the real part of the impedance-the conductivity or inversely the resistance. We suspect that even greater information will be available when both the real and imaginary parts are recovered.

In order to realize these measurements and report them back as a reconstructed image, the process of scanning impedance imaging requires three elements: (1) an impedance probe; (2) a conducting solution surrounding the material under test; and (3) a system for scanning precisely over a test sample. Because the method is a noncontact measurement technique, it can be used for nondestructive testing of a wide variety of materials. By immersing the probe in a conductive

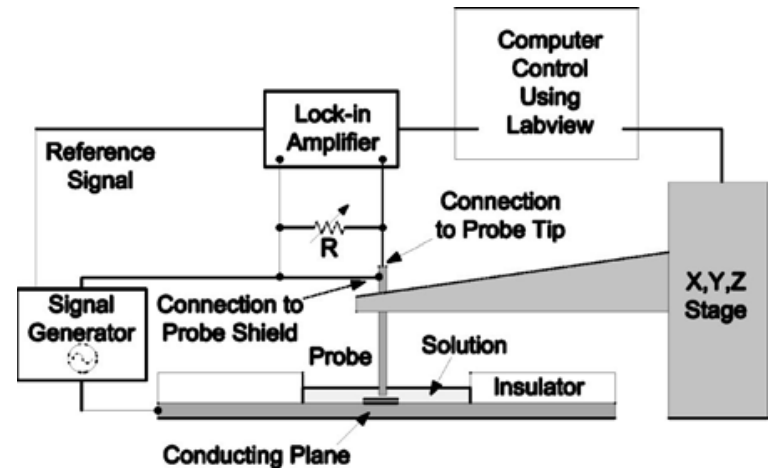

FIG. 1. Outline of scanning impedance imaging system.

solution, we also have the flexibility for innovative probe design without the constraint of making reliable electrical contacts directly between the probe and the material under test.

The realization of a scanning impedance imaging system is illustrated in Fig. 1. As shown in the figure, the real part of the impedance (resistance) of a sample was measured between the end of a probe and a conducting plane. Water with resistivity of $10 \mathrm{k} \Omega \mathrm{cm}$ filled in the space between the sample and the probe. A Newmark Systems Model NLS44-16 XYZ stage controller was used to move the probe, which has a minimum step increment of $10 \mu \mathrm{m}$. A programmable frequency generator was used to generate an ac signal. For all test results shown in this paper, a $2.5 \mathrm{~V}$ peak-to-peak, $10 \mathrm{kHz}$ ac signal was used to drive current through the probe and sample under test with a $27 \mathrm{k} \Omega$ resistor $(R)$ used for conversion to a voltage signal. Different resistances were tried but $27 \mathrm{k} \Omega$ was chosen because it produced the optimum resolution for our smallest geometry probe. A lock-in amplifier was used to measure voltage across the resistor and referenced to the signal generator. For the results reported here, the use of a lock-in amplifier was not necessary because the signal-to-noise ratios produced in the system were very high. As probe geometry shrinks in the future and signal magnitude decreases and as we evaluate phase shifts in return signals, this amplifier will likely be useful however. The ac drive voltage could also be lowered from $2.5 \mathrm{~V}$ to hundreds of millivolts in the current configuration without a significant loss in signal-to-noise ratio. This could be significant if it were desirable to minimize voltage across a sample like a cell wall or thin tissue. Finally, a computer running LABVIEW controlled probe movement, data gathering, and storage.

Of course the most critical component in the imaging system is the impedance probe. Impedance measurements were drastically different depending on the probe used. As illustrated by Hawkins et al. ${ }^{4}$ the resolution and signal-tonoise ratio is greatly enhanced if the probe is based on an inner conductor surrounded by an outer conductor as illustrated in Fig. 2(a). The idea behind this design is to bias the outer conductor at a higher voltage than the inner conductor, preventing unwanted current flux generated over the entire surface of the bottom conducting plane from being directed toward the inner conductor. The inner conductor called the "tip" of diameter $D$ is separated from the outer conductor or "shield" by an insulator. The spacing between the tip and the 


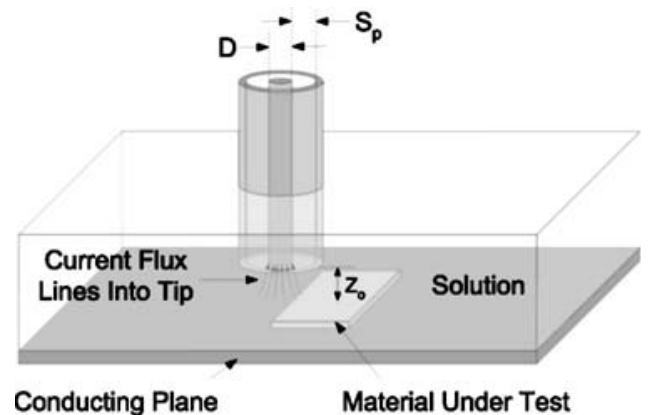

(a)

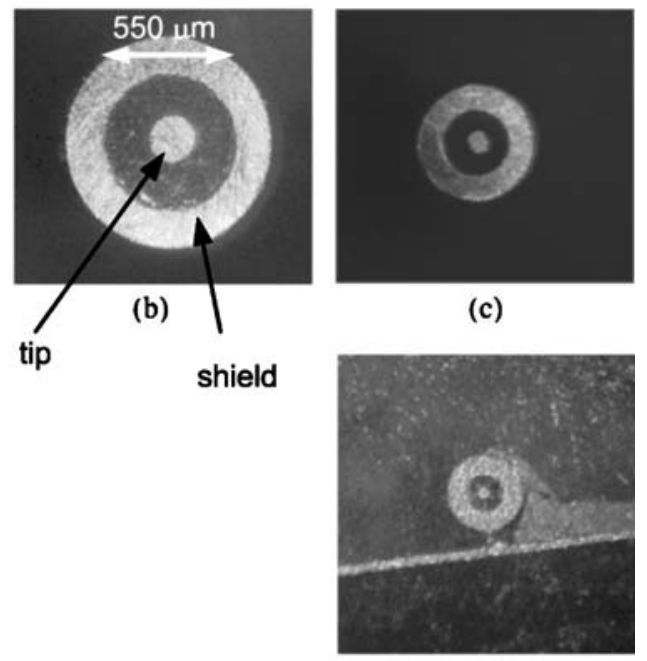

(d)

FIG. 2. Close up view of probe. (a) Illustration of probe lowered into solution and positioned near the material under test. (b)-(d) Microscope views of the cross sections at the end of real probes indicating tip and shield geometries: (b) $180 \mu \mathrm{m}$ tip, $550 \mu \mathrm{m}$ shield inner diameter, (c) $100 \mu \mathrm{m}$ tip, $300 \mu \mathrm{m}$ shield inner diameter, and (d) $30 \mu \mathrm{m}$ tip, $100 \mu \mathrm{m}$ shield inner diameter.

shield is given by $S_{p}$ as illustrated in Fig. 2(a). When used in the imaging system illustrated in Fig. 1, the resistor $R$ ensures that the shield is at a higher voltage potential than the tip so that current flux to the tip can come only from an area beneath the probe of total diameter less than or equal to the tip diameter $(D)$ plus twice the shield spacing $\left(S_{p}\right)$.

Small diameter shielded probes were constructed using insulated copper wire with metal diameters of 30,100 , and $180 \mu \mathrm{m}$. The wire was threaded into a stainless steel tube of inner diameters of 100,300 , and $550 \mu \mathrm{m}$, respectively, and embedded in nonconductive epoxy. The stainless steel tube served as the shield and the metal core of the wire the tip for the probes. The intention was to keep the diameter $D$ equal to the shield spacing $S_{p}$ as the probe sizes were scaled. Figures 2(b)-2(d) show digital photographs taken through an optical microscope showing the cross section of constructed probes.

A simple test was used to evaluate the performance of the constructed probes, as illustrated in Fig. 3. A thin insulator was placed on top of the conducting plane in our imaging system. The probe was then inserted into the system and positioned above the insulator. The probe was then moved from a position directly over the insulator to one directly over the uncovered conducting plane in small scan incre-

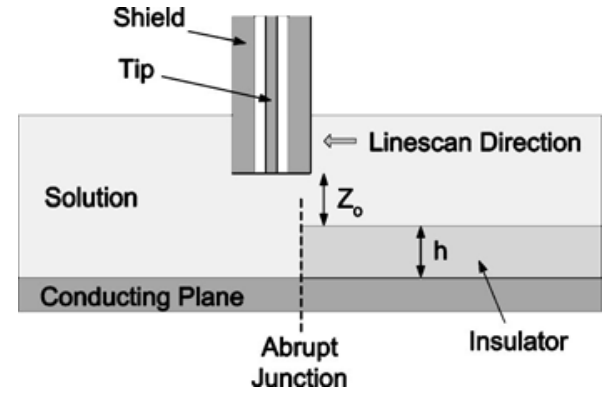

FIG. 3. Illustration of line scan measurement used to characterize probe resolution in scanning impedance imaging system.

ments with the current through the tip measured at each increment. The resulting line scan indicates the probe's ability to respond to the drastic change in impedance provided by the insulator. For very high resolution, a very sharp change in current would be expected at the abrupt junction created by the insulator. The automated XYZ stage allows for making line scan measurements at controlled variations of height above the insulator.

\section{EXPERIMENTAL RESULTS}

The types of line scans that are produced from the scanning impedance imaging system are shown in Fig. 4. These are the results for the $30 \mu \mathrm{m}$ (tip diameter) probe over a $170 \mu \mathrm{m}$ thick glass slide. The figure indicates line scans for different distances $Z_{o}$ above the glass. Plotted is the normalized rms voltage difference for the scan, meaning the measured voltage at a given point minus the minimum measured voltage for the entire line scan, all divided by the difference between the maximum voltage for the line scan minus the minimum voltage for the line scan. Voltage maximums occur when the probe is located over the conducting plane and current is greatest, while voltage minimums occur when the probe is located over the insulator and very little current flows. Normalizing the measured voltage in this way limits the graph extents between 0 and 1 . All of the scans shown in this plot are for the case when the shield of the probe is connected to the voltage source. When the shield is disconnected, the signal-to-noise ratio for the measured voltage sig-

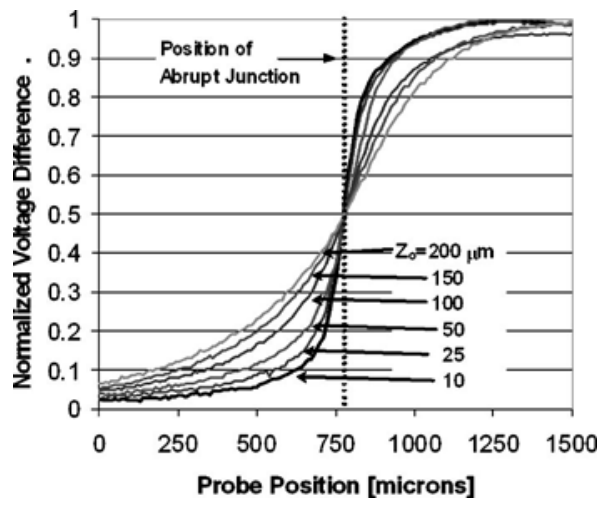

FIG. 4. Measured results of line scans using a $170 \mu \mathrm{m}$ thick glass slide for an insulator and a 30 micron diameter probe tip. The normalized voltage difference as a function of probe position for various probe heights is shown. The total scan length for these line scans was $3 \mathrm{~mm}$, but only $1.5 \mathrm{~mm}$ of the scan is shown, centered at the abrupt boundary. 
nal is much lower and the resolution worse. As expected, when the probe is closer to the insulator the resolution improves and the resulting response to the insulator is much sharper.

Line scan plots like those shown in Fig. 4 were made for the three probe sizes outlined in the previous section for two different insulators-a $20 \mu \mathrm{m}$ thick mica and a $170 \mu \mathrm{m}$ thick glass slide. Both insulators had extremely high impedances. To compare the performance of the probes, the distance between the points with $30 \%$ and $70 \%$ of the maximum voltage for the line scans is plotted versus probe height $Z_{o}$. This translates to how effectively the probe can indicate the abrupt change in impedance created by the insulator. The smaller the $30 \%-70 \%$ distance, the better the resolution for a particular probe and scan condition.

Figure 5(a) shows the measured $30 \%-70 \%$ distance when the glass slide was used as the insulator, while Fig. 5(b) shows the same quantity when the mica was used. As indicated by these figures, the best resolution results when the smallest diameter tip is used to probe the thinnest insulating sample. The minimum resolution measured was $29.5 \mu \mathrm{m}$ for a $30 \mu \mathrm{m}$ probe tip over $20 \mu \mathrm{m}$ thick mica. The figure also indicates that although image resolution improves when scaling down probe diameter, strictly speaking, ultimate resolution does not scale linearly simply with tip diameter, i.e., by using a $1 \mu \mathrm{m}$ diameter tip, one cannot hope to obtain a $1 \mu \mathrm{m}$ resolution when measuring a $20 \mu \mathrm{m}$ thick sample. To understand better what is happening, the data was fit to a low frequency, linear model where the current and conductivity are related by a convolution. ${ }^{8}$ The convolution uses a blurring function that takes into account the distance $\left(t=h+Z_{o}\right)$ between the tip and the ground plane, and the results are useful mostly for predicting trends for various parameters. This model results in two simplified equations valid in different regimes. The first is valid when the tip diameter $(D)$ is much smaller than the height and the blurring is dominated by the separation between the probe and the insulator. In this case the one-dimensional step response implied by this blurring model is

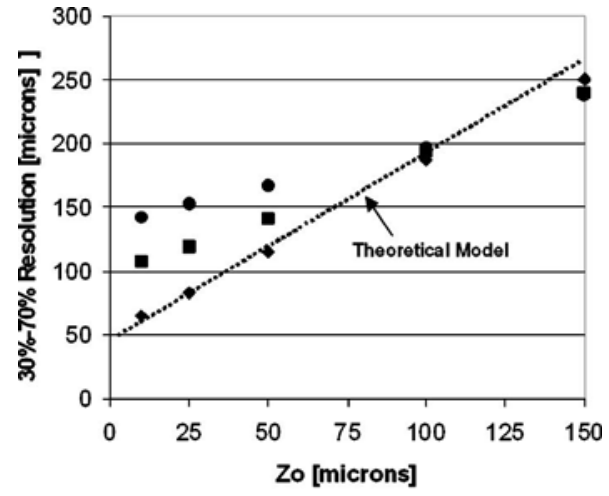

(a)

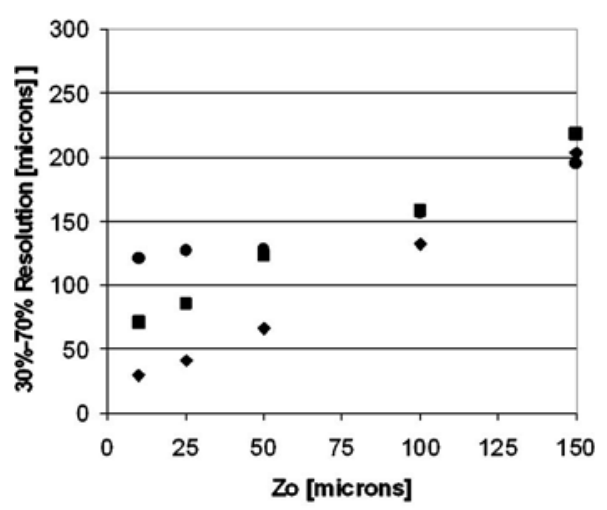

(b)

FIG. 5. The resolution (defined as the distance between the $30 \%$ and $70 \%$ of the maximum voltage difference) as a function of probe height for various probes and insulators. (a) Insulator was $170 \mu \mathrm{m}$ thick glass slide, (diamonds) correspond to a $30 \mu \mathrm{m}$ tip probe, (squares) $100 \mu \mathrm{m}$ tip probe, and (circles) $180 \mu \mathrm{m}$ tip probe. The theoretical prediction for the slope between resolution distance and $Z_{o}$ is shown by the dotted line. (b) Insulator was $20 \mu \mathrm{m}$ thick mica, (diamonds) correspond to a $30 \mu \mathrm{m}$ tip probe, (squares) $100 \mu \mathrm{m}$ tip probe, and (circles) $180 \mu \mathrm{m}$ tip probe.

$$
s(x)=\left[\frac{1}{2}+\frac{1}{\pi} \arctan \left(\frac{x}{t}\right)\right] .
$$

The second case is when $t \ll D$ in which case the tip width will dominate the blurring and the one-dimension stepresponse becomes

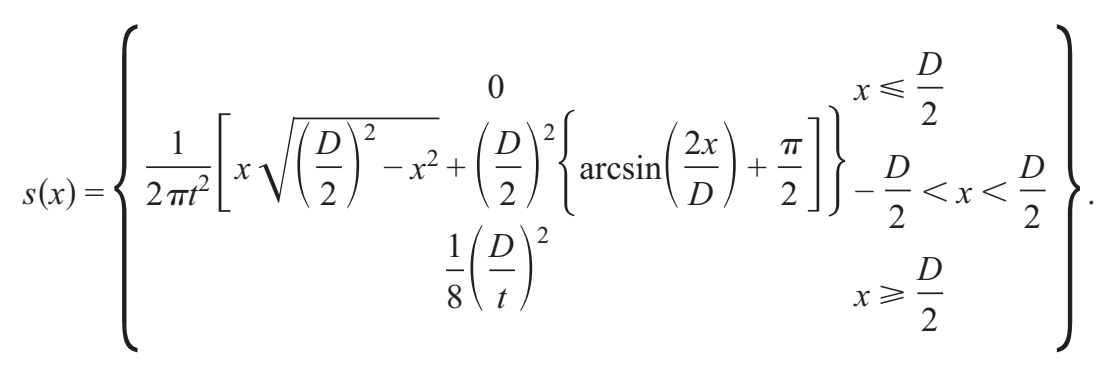

For the first case (large $t$ compared to D), Eq. (2) indicates that the slope of the $30 \%$ to $70 \%$ resolution distance versus $Z_{o}$ should be approximately 1.45 . The measurement case that best fits this scenario is the $30 \mu \mathrm{m}$ probe and the $170 \mu \mathrm{m}$ thick insulator. In this case the calculated best linear slope fit to the measured data is 1.34 , quite close to the predicted 
slope of 1.45. The predicted slope based on the model is shown in Fig. 5(a). As the ratio of tip diameter to total distance from the conducting plane increases, the slope for the measured data should decrease and look less and less linear. This trend is certainly verified by the data in Fig. 5. For case (2), corresponding to Eq. (3), when $t \ll D$, the $30 \%-70 \%$ resolution should remain approximately constant with increasing $Z_{o}$. This is shown by the data that best fits this scenario where the tip is $180 \mu \mathrm{m}$ in diameter, the insulator is $20 \mu \mathrm{m}$ thick, and the probe is very close to the insulator. As shown in Fig. 5(b), there is very little change in the measured resolution for the $180 \mu \mathrm{m}$ tip when $Z_{o} \leqslant 50 \mu \mathrm{m}$.

Based on the measured data and how it matches our simple physical model for blurring, some firm conclusions about resolution can be made. Ultimate resolution will be determined both by probe size and sample thickness. As shown in Fig. 5(a), 30\%-70\% resolution can be better than the sample thickness if the probe has a small enough diameter, but submicron resolution is not possible for a $200 \mu \mathrm{m}$ thick sample no matter how small the size of the probe. If the sample thickness is about one micron however, it appears that submicron resolution would be possible even without image reconstruction given a probe with tip diameter less than one micron. The fabrication of single micron and submicron capable probes, will most likely require the use of semiconductor microfabrication techniques-where such feature sizes are readily achieved. This work is currently underway.

Blurring and ultimate image resolution can also be affected by the resistivity of the aqueous solution. Higher resistivity solutions would tend to produce lower image resolutions given the same probe geometry and height $Z_{o}$, because the current flux would spread out more rapidly for a given distance above the conducting plane.

In addition to line scans, the SII system was used to produce full two-dimensional impedance maps for different materials to provide a more concrete view of its imaging capability. Figure 6 shows the impedance map of a $170 \mu \mathrm{m}$ thick piece of glass sitting on an aluminum coated silicon

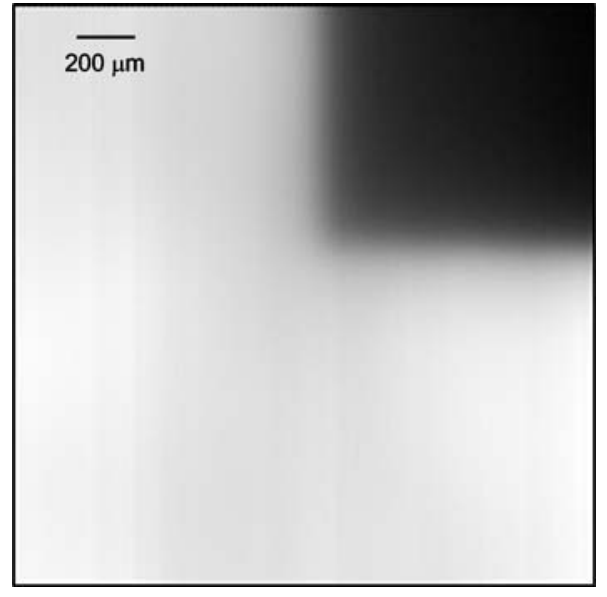

FIG. 6. An impedance image of the corner of a $170 \mu \mathrm{m}$ micron thick piece of glass sitting on an aluminum ground plane. The scan was produced using a probe with a $30 \mu \mathrm{m}$ diameter probe tip. The step increment for the image was $10 \mu \mathrm{m}$ in both the $x$ and $y$ directions. The probe was approximately $10 \mu \mathrm{m}$ above the mica's top surface. The glass slide is located in the upper right hand corner of the figure and appears dark in the impedance image.

wafer. Coating the silicon wafer provides a very flat ground plane for reference. The $30 \mu \mathrm{m}$ diameter probe tip was used to create this image. In this figure, measured voltage difference is plotted compared to the minimum voltage so that the glass appears dark compared to the uncovered aluminum areas. As can be seen, a high contrast image is produced as might be hoped for an insulating material.

${ }^{1}$ S. Kalaitzidis and K. Christanis, Int. J. Coal Geol. 54, 69 (2003).

${ }^{2}$ J. M. Kim, T. Ohtani, and H. Muramatsu, Surf. Sci. 549, 273 (2004).

${ }^{3}$ R. Shao, S. V. Kalinin, and D. A. Bonnell, Appl. Phys. Lett. 82, 1869 (2003)

${ }^{4}$ A. R. Hawkins, H. Liu, T. E. Oliphant, and S. M. Schultz, Appl. Phys. Lett. 85, 1080 (2004)

${ }^{5}$ F. C. Grant, J. Am. Med. Assoc. 81, 2166 (1923).

${ }^{6}$ V. A. Cherepenin, A. Y. Karpov, A. V. Korjenevsky, V. N. Komienko, Y. S. Kultiasov, M. B. Ochapkin, O. V. Trochanova, and J. D. Meister, IEEE Trans. Med. Imaging 21, 662 (2002).

${ }^{7}$ L. A. Geddes and L. E. Baker, Med. Biol. Eng. 5, 271 (1967).

${ }^{8}$ T. E. Oliphant, H. Liu, T. Shang, S. M. Schultz, and A. R. Hawkins (unpublished). 